Homology, Homotopy and Applications, vol.20(2), 2018, pp.341-360

\title{
ITERATED DOUBLES OF THE JOKER AND THEIR REALISABILITY
}

\author{
ANDREW BAKER
}

(communicated by Donald M. Davis)

\begin{abstract}
Let $\mathcal{A}(1)^{*}$ be the subHopf algebra of the mod 2 Steenrod algebra $\mathcal{A}^{*}$ generated by $\mathrm{Sq}^{1}$ and $\mathrm{Sq}^{2}$. The Joker is the cyclic $\mathcal{A}(1)^{*}$-module $\mathcal{A}(1)^{*} / \mathcal{A}(1)^{*}\left\{\mathrm{Sq}^{3}\right\}$ which plays a special rôle in the study of $\mathcal{A}(1)^{*}$ modules. We discuss realisations of the Joker both as an $\mathcal{A}^{*}$-module and as the cohomology of a spectrum. We also consider analogous $\mathcal{A}(n)^{*}$-modules for $n \geqslant 2$ and prove realisability results (both stable and unstable) for $n=2,3$ and non-realisability results for $n \geqslant 4$.
\end{abstract}

\section{Introduction}

The cyclic $\mathcal{A}(1)^{*}$-module $\mathcal{A}(1)^{*} / \mathcal{A}(1)^{*}\left\{\mathrm{Sq}^{3}\right\}$, commonly known as the Joker, was shown by Adams and Priddy [2] to give rise to a torsion summand in the Picard group of invertible stable $\mathcal{A}(1)^{*}$-modules. Here is a representation of the Joker where a vertical line indicates the action of $\mathrm{Sq}^{1}$ and a curved line indicates the action of $\mathrm{Sq}^{2}$.

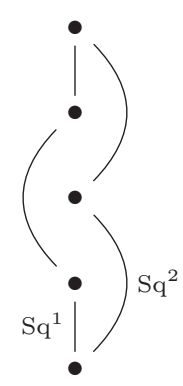

There are various choices for the grading, but for topological reasons we use the one where the lowest degree of a nontrivial element is 0. More details about the Joker and its homological algebra can be found in [5, Appendix A.8]. For a recent result which highlights the special significance of the Joker see [4]. Incidentally, the use of the name Joker appears to be due to Frank Adams, although the earliest published occurrence that we are aware of is in [10]; it may be based on the similarity of the diagram above to a traditional jester's hat.

The mathematics in this paper owes much to the insights and inspiration of Michael Barratt and Mark Mahowald and I would like to dedicate it to their memory.

Received March 25, 2018, revised April 26, 2018; published on July 11, 2018.

2010 Mathematics Subject Classification: Primary 55P42; Secondary 55S10, 55S20.

Key words and phrases: stable homotopy theory, Steenrod algebra.

Article available at http://dx.doi.org/10.4310/HHA.2018.v20.n2.a17

Copyright (C) 2018, Andrew Baker. Permission to copy for private use granted. 
The $\mathcal{A}(1)^{*}$-module structure of the Joker extends in two ways to an $\mathcal{A}^{*}$-module structure determined by whether $\mathrm{Sq}^{4}$ acts non-trivially or not between the bottom and top degrees. The resulting $\mathcal{A}^{*}$-modules are linear duals of each other. We will show that both can be realised as cohomology of finite CW spectra which are SpanierWhitehead dual using a construction we learnt from Peter Eccles, however, it also appeared in Mike Hopkins' Oxford PhD thesis but seems not to be otherwise published. We will show in Theorem 5.1 that these can be realised as cohomology of spaces with bottom cells of degrees 2 and 4, respectively.

Using a construction based on doubling, we introduce higher versions of the Joker defined as cyclic $\mathcal{A}(n)^{*}$-modules and show that these can be realised as cohomology of spectra precisely when $n \leqslant 3$. Most cases of the non-realisability result can be verified by a direct application of Adams' result on Hopf invariant 1, however, in one case we resort to a more delicate argument using the precise form of his factorisation of $\mathrm{Sq}^{2^{r}}$ for $r \geqslant 4$, so we give a proof which applies in all cases. In the cases where we can realise these modules, our constructions depend on the existence of triple Toda brackets containing the first three elements of Kervaire invariant 1, i.e., $\eta^{2}, \nu^{2}, \sigma^{2}$. Finally, we consider unstable realisations and show that for $n=1,2$ we can indeed realise optimal unstable versions of the higher Joker modules; the techniques used involve modifying naturally occurring spaces by mapping into Eilenberg-Mac Lane spaces and certain spaces in the spectra $k \mathrm{O}$ and tmf, thus giving alternatives to the stable constructions above.

For the convenience of the reader, we include a brief appendix in which some connectivity results on infinite loop spaces are given; this material is standard but we were unable to locate convenient references.

We also make numerous references to calculational results obtained using the Adams spectral sequence. The reader can find relevant charts in the earlier arxiv versions of this paper

$$
\text { https://arxiv.org/abs/1710.02974 }
$$

but at the request of the Editor we have omitted most of these. They were obtained using Bob Bruner's programmes available at the following address.

$$
\text { http://www.math.wayne.edu/ rrb/cohom/index.html }
$$

\section{Acknowledgments}

I would like to thank the following for helpful comments and insights: Bob Bruner and John Rognes (from whom I learnt an enormous amount about working with the Steenrod algebra), Don Davis, Peter Eccles (who showed me how to use Toda brackets to construct complexes efficiently and so initiated the work described) and Grant Walker.

\section{Conventions \& notations}

Throughout we work locally at the prime 2 .

To avoid excessive display of gradings we will often suppress cohomological degrees and write $V$ for a cohomologically graded vector space $V^{*}$; in particular, we will 
often write $\mathcal{A}$ for the Steenrod algebra. The linear dual of $V$ is $\mathrm{D} V$ where $(\mathrm{D} V)^{k}=$ $\operatorname{Hom}_{\mathbb{F}_{2}}\left(V^{-k}, \mathbb{F}_{2}\right)$, and we write $V[m]$ for graded vector space with $(V[m])^{k}=V^{k-m}$, so for the cohomology of a spectrum $X, H^{*}\left(\Sigma^{m} X\right)=H^{*}(X)[m]$.

For a connected graded algebra $\mathcal{B}^{*}$ we will write $\mathcal{B}^{+}$for its positive degree part.

We will denote the Spanier-Whitehead dual of a spectrum $X$ by $D X$.

\section{1. $\mathcal{A}$-module structures on the Joker and duality}

The Joker has two possible $\mathcal{A}$-module structures corresponding to the choice of action of $\mathrm{Sq}^{4}$ between the top and bottom degrees. The resulting $\mathcal{A}$-modules Joker $_{0}^{*}$ and Joker $_{1}^{*}$ are displayed in the following diagrams in which the shorter edges represent non-trivial $\mathrm{Sq}^{1}$ and $\mathrm{Sq}^{2}$ actions.

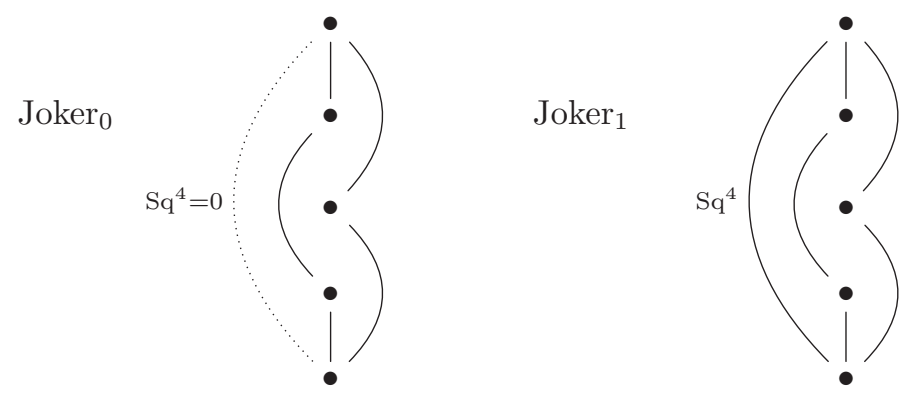

Recall that for a left $\mathcal{A}$-module $M$, the $\mathbb{F}_{2}$-linear dual $\mathrm{D} M$ is naturally a right $\mathcal{A}$-module where for $f \in \mathrm{D} M, \theta \in \mathcal{A}$ and $x \in M$,

$$
(f \cdot \theta)(x)=f(\theta x)
$$

There is an associated left module structure given by

$$
(\theta \cdot f)(x)=(f \cdot \chi \theta)(x)=f(\chi \theta x),
$$

where $\chi: \mathcal{A} \rightarrow \mathcal{A}$ is the antipode. For a finite $\mathrm{CW}$ complex spectrum $Z$, as a left $\mathcal{A}$-module the cohomology of the Spanier-Whitehead dual $D Z$ satisfies

$$
H^{*}(D Z) \cong \mathrm{D}\left(H^{*}(Z)\right) \text {. }
$$

Since the following relations hold in $\mathcal{A}$,

$$
\begin{aligned}
& \mathrm{Sq}^{3}=\mathrm{Sq}^{1} \mathrm{Sq}^{2}, \quad \mathrm{Sq}^{2} \mathrm{Sq}^{2}=\mathrm{Sq}^{1} \mathrm{Sq}^{2} \mathrm{Sq}^{1}, \\
& \chi \mathrm{Sq}^{1}=\mathrm{Sq}^{1}, \quad \chi \mathrm{Sq}^{2}=\mathrm{Sq}^{2}, \\
& \chi \mathrm{Sq}^{4}=\mathrm{Sq}^{4}+\mathrm{Sq}^{1} \mathrm{Sq}^{2} \mathrm{Sq}^{1}=\mathrm{Sq}^{4}+\mathrm{Sq}^{2} \mathrm{Sq}^{2},
\end{aligned}
$$

it follows that Joker $_{0}$ and Joker $_{1}$ are dual up to a degree shift, i.e.,

$$
\text { Joker }_{1} \cong \operatorname{DJoker}_{0}[4] \text {. }
$$

\section{Doubling and higher versions of the Joker}

Doubling for $\mathcal{A}$ and $\mathcal{A}(n)$ are discussed in Margolis [14, Section 15.3] (for a particularly relevant result on modules see Theorem 31 ). We give a brief description and, in particular, explain what happens under iterated doubling. 
The dual of $\mathcal{A}(n)$ is the quotient Hopf algebra

$$
\begin{aligned}
\mathcal{A}(n)_{*} & =\mathcal{A}_{*} /\left(\zeta_{1}^{2^{n+1}}, \zeta_{2}^{2^{n}}, \ldots, \zeta_{n+1}^{2}, \zeta_{n+2}, \ldots\right) \\
& =\mathbb{F}_{2}\left[\overline{\zeta_{1}}, \overline{\zeta_{2}}, \ldots, \overline{\zeta_{n+1}}\right] /\left({\overline{\zeta_{1}}}^{2 n+1}, \bar{\zeta}^{2}, \ldots,{\overline{\zeta_{n+1}}}^{2}\right),
\end{aligned}
$$

where $\overline{(-)}$ indicates residue class. The dual of the normal exterior subHopf algebra $\mathcal{E}(n) \subseteq \mathcal{A}(n)$ generated by the Milnor primitives $\mathrm{P}_{t}^{0}(1 \leqslant t \leqslant n+1)$ is the quotient exterior algebra

$$
\left.\mathcal{E}(n)_{*}=\mathcal{A}_{*} /\left(\zeta_{1}^{2}, \zeta_{2}^{2}, \ldots, \zeta_{n+1}^{2}, \zeta_{n+2}, \zeta_{n+3}, \ldots\right) \cong \mathcal{A}(n)_{*} /{\overline{\zeta_{1}}}^{2}, \bar{\zeta}_{2}^{2}, \ldots, \bar{\zeta}_{n+1}^{2}\right) .
$$

The dual of the quotient Hopf algebra

$$
\mathcal{A}(n) / / \mathcal{E}(n)=\mathcal{A}(n) \otimes_{\mathcal{E}(n)} \mathbb{F}_{2} \cong \mathcal{A}(n) / \mathcal{A}(n) \mathcal{E}(n)^{+}
$$

is

$$
\left.\mathcal{A}(n)_{*} \square_{\mathcal{E}(n)_{*}} \mathbb{F}_{2}=\mathbb{F}_{2}\left[{\overline{\zeta_{1}}}^{2},{\overline{\zeta_{2}}}^{2}, \ldots,{\overline{\zeta_{n+1}}}^{2}\right] /{\overline{\zeta_{1}}}^{2 n+1},{\overline{\zeta_{2}}}^{2}, \ldots,{\overline{\zeta_{n+1}}}^{2}\right) \subseteq \mathcal{A}(n)_{*}
$$

There is an external Frobenius homomorphism $\mathbf{f}: \mathcal{A}(n)_{*} \rightarrow \mathcal{A}(n+1)_{*}$ which factors through the dual of $\mathcal{A}(n) / / \mathcal{E}(n)$ and satisfies $\mathbf{f}\left(\overline{\zeta_{r}}\right)={\overline{\zeta_{r}}}^{2}$.

$$
\mathcal{A}(n)_{*} \longrightarrow \mathcal{A}(n+1)_{*} \square_{\mathcal{E}(n+1)_{*}} \mathbb{F}_{2}
$$

More generally there are iterations $\mathbf{f}^{(k)}: \mathcal{A}(n)_{*} \rightarrow \mathcal{A}(n+k)_{*}$ where $k \geqslant 0$, so that $\mathbf{f}^{(0)}=\mathbf{f}$ and $\mathbf{f}^{(k)}\left(\overline{\zeta_{r}}\right)={\overline{\zeta_{r}}}^{2 k}$.

$$
\mathcal{A}(n)_{*} \longrightarrow \mathcal{A}(n+k)_{*} \square_{\mathcal{E}(n+k)_{*}} \mathbb{F}_{2}
$$

Each $\mathbf{f}^{(k)}$ is clearly a Hopf algebra homomorphism and there is a dual Verschiebung Hopf algebra homomorphism $\mathbf{v}^{(k)}: \mathcal{A}(n+k) \rightarrow \mathcal{A}(n)$.

$$
\mathcal{A}(n) \longleftarrow \mathbf{v}^{(k)} \mathcal{A}(n+k) / / \mathcal{E}(n+k)
$$

Since

$$
\mathbf{f}^{(k)}\left(\overline{\xi_{1}^{i_{1}} \cdots \xi_{\ell}^{i_{\ell}}}\right)=\overline{\xi_{1}^{2^{k} i_{1}} \cdots \xi_{\ell}^{2^{k} i_{\ell}}}
$$

the effect of $\mathbf{v}^{(k)}$ is easily seen using the Milnor basis dual to the monomial basis in the elements $\overline{\xi_{r}}=\chi \overline{\zeta_{r}}$ and we have

$$
\mathbf{v}^{(k)}\left(\operatorname{Sq}\left(j_{1}, \ldots, j_{\ell}\right)\right)= \begin{cases}\operatorname{Sq}\left(j_{1}^{\prime}, \ldots, j_{\ell}^{\prime}\right) & \text { if } j_{r}=2^{k} j_{r}^{\prime} \text { for all } r \\ 0 & \text { otherwise. }\end{cases}
$$


Since $\operatorname{Sq}(j)=\mathrm{Sq}^{j}$

$$
\mathbf{v}^{(k)}\left(\mathrm{Sq}^{j}\right)= \begin{cases}\mathrm{Sq}^{j^{\prime}} & \text { if } j=2^{k} j^{\prime} \\ 0 & \text { otherwise }\end{cases}
$$

Finally, for $1 \leqslant t \leqslant n+1$ the elements $\mathbf{v}^{(k)}\left(\mathrm{P}_{t}^{s}\right) \in \mathcal{A}(n)$ are given by

$$
\mathbf{v}^{(k)}\left(\mathrm{P}_{t}^{s}\right)=\mathbf{v}^{(k)}\left(\mathrm{Sq}(\overbrace{0, \ldots, 0,2^{s}}^{t})\right)= \begin{cases}\mathrm{P}_{t}^{s-k} & \text { if } s \geqslant k, \\ 0 & \text { otherwise. }\end{cases}
$$

Let ${ }_{\mathcal{A}(n)} \mathcal{M}^{(r)}$ be the full subcategory of ${ }_{\mathcal{A}(n)} \mathcal{M}$ consisting of modules concentrated in degrees divisible by $2^{r}$; for example ${ }_{\mathcal{A}(n)} \mathcal{M}^{(0)}={ }_{\mathcal{A}(n)} \mathcal{M}$, and ${ }_{\mathcal{A}(n)} \mathcal{M}^{(1)}={ }_{\mathcal{A}(n)} \mathcal{M}^{\text {ev }}$. The Verschiebung $\mathbf{v}^{(k)}$ together with the quotient homomorphism $\pi: \mathcal{A}(n+k) \rightarrow$ $\mathcal{A}(n+k) / / \mathcal{E}(n+k)$ induces restriction functors between categories of left modules fitting into the following commutative diagram.

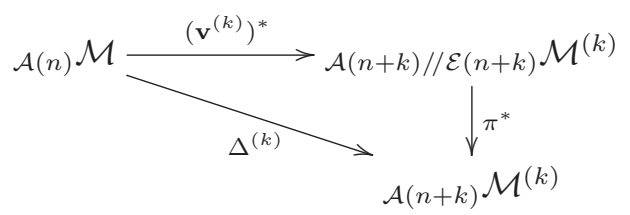

Here $\Delta^{(k)}$ multiplies degrees by $2^{k}$ and it is a monoidal functor since $\mathbf{v}^{(k)}$ and $\pi$ are both homomorphisms of Hopf algebras.

By $\left[\mathbf{1 4}\right.$, Theorem 15.3.31] $\Delta^{(1)}$ is an isomorphism of categories, but when $k>1$ this is not true. However, this can be corrected by replacing $\mathcal{A}(n+k) / / \mathcal{E}(n+k)$ by the quotient of $\mathcal{A}(n+k)$ by the ideal generated by a larger set of the elements $\mathrm{P}_{t}^{s}$. Let

$$
\mathcal{E}(n+k, k)=\mathbb{F}_{2}\left(\mathrm{P}_{t}^{s}: 1 \leqslant t \leqslant n+k+1,0 \leqslant s<k\right) \subseteq \mathcal{A}(n+k)
$$

and consider the normal quotient

$$
\mathcal{A}(n+k) / / \mathcal{E}(n+k, k)=\mathcal{A}(n+k) / \mathcal{A}(n+k) \mathcal{E}(n+k, k)^{+} \cong \mathcal{A}(n+k) \otimes_{\mathcal{E}(n+k, k)} \mathbb{F}_{2} .
$$

Then (2.1) can be replaced by

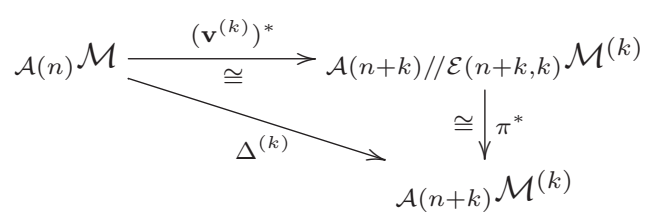

so that the proof of Margolis still applies to show that this $\Delta^{(k)}$ is an isomorphism of categories.

We remark that $\Delta^{(k)}$ does not induce a functor on stable module categories since it does not preserve projective modules.

For each $n \geqslant 2$, iterated doubling gives a generalisation of the Joker to a cyclic $\mathcal{A}(n)$-module $\operatorname{Joker}(n)=\Delta^{(n-1)}$ (Joker). The actions of $\mathrm{Sq}^{2^{n-1}}$ and $\mathrm{Sq}^{2^{n}}$ on $\operatorname{Joker}(n)$ 
are shown below.
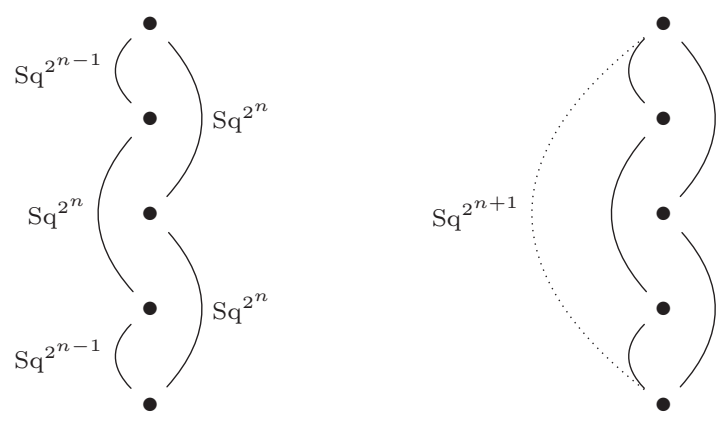

There are two extensions to $\mathcal{A}$-module structures, each determined by a choice of action by $\mathrm{Sq}^{2^{n+1}}$ from the top to the bottom degree, and we denote the resulting $\mathcal{A}$-modules by $\operatorname{Joker}(n)_{0}$ and $\operatorname{Joker}(n)_{1}$ depending on whether $\mathrm{Sq}^{2^{n+1}}$ acts trivially or not. It is straightforward to verify that the action of $\chi \mathrm{Sq}^{2^{n+1}}$ on $\operatorname{Joker}(n)_{0}$ is non-trivial and

$$
\operatorname{Joker}(n)_{1} \cong \operatorname{DJoker}(n)_{0}\left[2^{n+1}\right] .
$$

As an $\mathcal{A}(n)$-module, Joker $(n)$ is finitely presented. For example, Joker(1) has minimal presentation

$$
0 \leftarrow \operatorname{Joker}(1) \leftarrow \mathcal{A}(1) \leftarrow \mathcal{A}(1)[3]
$$

while

$$
\operatorname{Joker}(2)=\mathcal{A}(2) / \mathcal{A}(2)\left\{\mathrm{P}_{1}^{0}, \mathrm{P}_{2}^{0}, \mathrm{P}_{3}^{0}, \mathrm{Sq}^{6}\right\}=\mathcal{A}(2) / \mathcal{A}(2)\left\{\mathrm{P}_{1}^{0}, \mathrm{P}_{2}^{0}, \mathrm{Sq}^{6}\right\},
$$

so it has a minimal presentation

$$
0 \leftarrow \operatorname{Joker}(2) \leftarrow \mathcal{A}(2) \leftarrow \mathcal{A}(2)[1] \oplus \mathcal{A}(2)[3] \oplus \mathcal{A}(2)[6] .
$$

Finally,

$$
\operatorname{Joker}(3)=\mathcal{A}(3) / \mathcal{A}(3)\left\{\mathrm{P}_{1}^{0}, \mathrm{P}_{2}^{0}, \mathrm{P}_{3}^{0}, \mathrm{P}_{1}^{1}, \mathrm{P}_{2}^{1}, \mathrm{Sq}^{12}\right\}=\mathcal{A}(3) / \mathcal{A}(3)\left\{\mathrm{P}_{1}^{0}, \mathrm{P}_{1}^{1}, \mathrm{P}_{2}^{1}, \mathrm{Sq}^{12}\right\}
$$

and there is a minimal presentation

$$
0 \leftarrow \operatorname{Joker}(3) \leftarrow \mathcal{A}(3) \leftarrow \mathcal{A}(3)[1] \oplus \mathcal{A}(3)[2] \oplus \mathcal{A}(3)[6] \oplus \mathcal{A}(3)[12] .
$$

Of course the $\mathcal{A}$-modules $\operatorname{Joker}(n)_{0}$ and $\operatorname{Joker}(n)_{1}$ are not finitely presented. These presentations can be extended further using the Sage package of Mike Catanzaro and Bob Bruner which can be found at

$$
\text { http://www.math.wayne.edu/ mike/mods/ }
$$

and is documented in $[6]$.

\section{Some recollections on Toda brackets}

For ease of reference, we recall some basic ideas about triple Toda brackets in homotopy theory. A classic source for the basic ideas is the book of Mosher and 
Tangora [16] and Toda's seminal work [17] provides a more exhaustive account, while Cohen [7] gives a different treatment, also discussed by Whitehead [19].

Let

$$
W \stackrel{f}{\longrightarrow} X \stackrel{g}{\longrightarrow} Y \stackrel{h}{\longrightarrow} Z
$$

be a sequence of maps (of based spaces or spectra) and assume that $g f$ and $h g$ are null homotopic. The mapping sequence for $g$ extends to a commutative diagram of solid arrows

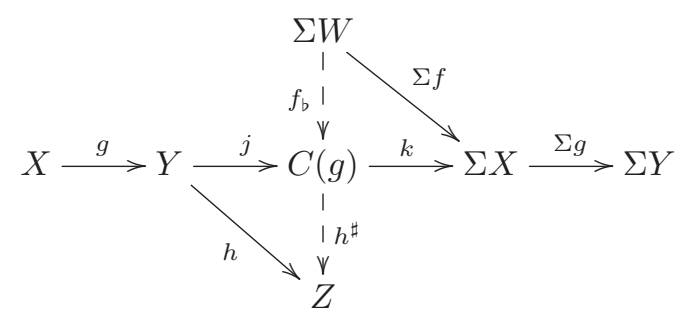

and the composition $h^{\sharp} f_{\mathrm{b}}: \Sigma W \rightarrow Z$ represents the Toda bracket $\langle f, g, h\rangle$. Of course this element is not necessarily well defined up to homotopy: the choices in $f_{\mathrm{b}}$ and $h^{\sharp}$ contribute indeterminacy subgroups $h_{*}[\Sigma W, Y]$ and $(\Sigma f)^{*}[\Sigma X, Y]$ and when $W$ is a suspension or a spectrum

$$
\operatorname{indet}\langle f, g, h\rangle=h_{*}[\Sigma W, Y]+(\Sigma f)^{*}[\Sigma X, Y],
$$

and

$$
\langle f, g, h\rangle=h^{\sharp} f_{\mathrm{b}}+h_{*}[\Sigma W, Y]+(\Sigma f)^{*}[\Sigma X, Y],
$$

for some given choice of $f_{\mathrm{b}}$ and $h^{\sharp}$.

Here are some important examples of such Toda brackets in the stable homotopy groups of spheres $\pi_{*}(S)$ where $S=S_{(2)}^{0}$ is the 2-local sphere spectrum. As usual, we identify $\theta \in \pi_{n}(S)$ with $\Sigma^{k} \theta \in \pi_{n+k}\left(\Sigma^{k} S\right) \cong \pi_{n+k}\left(S^{k}\right)$.

$$
\begin{aligned}
& \langle 2, \eta, 2\rangle=\left\{\eta^{2}\right\}, \\
& \langle\eta, \nu, \eta\rangle=\left\{\nu^{2}\right\}, \\
& \langle\nu, \sigma, \nu\rangle=\left\{\sigma^{2}\right\} .
\end{aligned}
$$

Of course these elements $\theta_{1}=\eta^{2}, \theta_{2}=\nu^{2}$ and $\theta_{3}=\sigma^{2}$ are the first elements of Kervaire invariant 1 .

Proof/justification. Using the Peterson-Stein formula [16] or performing calculations with Massey products in Ext $_{\mathcal{A}}$, it is straightforward to see that these brackets contain the stated elements; alternatively see [17, Corollary 3.7]. Also,

$$
\begin{aligned}
& \operatorname{indet}\langle 2, \eta, 2\rangle=2 \pi_{1}(S)=0, \\
& \operatorname{indet}\langle\eta, \nu, \eta\rangle=\eta \pi_{5}(S)=0,
\end{aligned}
$$

since $\pi_{1}(S) \cong \mathbb{Z}_{2}$ and $\pi_{5}(S)=0$. To see that $\operatorname{indet}\langle\nu, \sigma, \nu\rangle=0$, we need to consider 
the Adams spectral sequence

$$
\mathrm{E}_{2}^{s, t}=\operatorname{Ext}_{\mathcal{A}}^{s, t}\left(\mathbb{F}_{2}, \mathbb{F}_{2}\right) \Longrightarrow \pi_{t-s}(S)
$$

in degree 14. Although there is an element $h_{2} \mathrm{P} h_{2} \in \mathrm{E}_{2}^{6,20}$ this is killed by the differential $d_{3}$, so $\nu \pi_{11}(S)=0$.

\section{Constructing Joker spectra}

The main idea for this construction was explained to us by Peter Eccles, and it also appears in the unpublished Oxford PhD thesis of Mike Hopkins [11] (see Section 1.7). We will make use of the well-known Toda bracket $\langle 2, \eta, 2\rangle=\left\{\eta^{2}\right\} \subseteq \pi_{2}\left(S^{0}\right)$.

Let $2^{\sharp}: C(\eta) \rightarrow S^{0}$ extend 2 on the bottom cell; there is no indeterminacy in this choice because the non-trivial element $\eta^{2} \in \pi_{2}\left(S^{0}\right)$ is in the image of the map $\pi_{2}\left(S^{1}\right) \rightarrow \pi_{2}\left(S^{0}\right)$ induced by $\eta: S^{2} \rightarrow S^{1}$ on domains. Let $2_{b}: S^{2} \rightarrow C(\eta)$ be the coextension of the degree 2 map onto the top cell; again there is no indeterminacy in this choice since the non-trivial element $\eta^{2} \in \pi_{2}\left(S^{0}\right)$ is in the image of the map $\pi_{2}\left(S^{1}\right) \rightarrow \pi_{2}\left(S^{0}\right)$ induced by $\eta: S^{1} \rightarrow S^{0}$ on codomains.

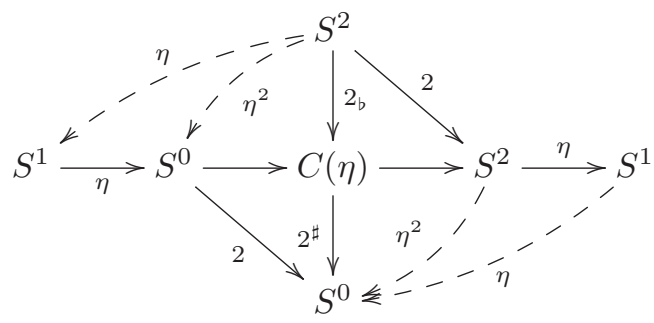

By (3.1a) the composition $\eta^{2}-2^{\sharp} \circ 2_{b}$ in the following diagram is null homotopic.

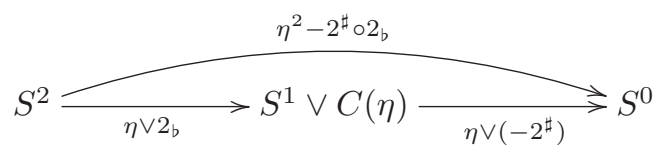

The mapping sequences for $\eta \vee 2^{\sharp}$ and $\eta \vee 2_{b}$ together yield the following diagram of solid and dashed arrows.
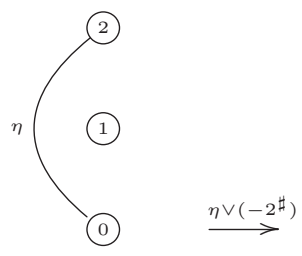

(0)
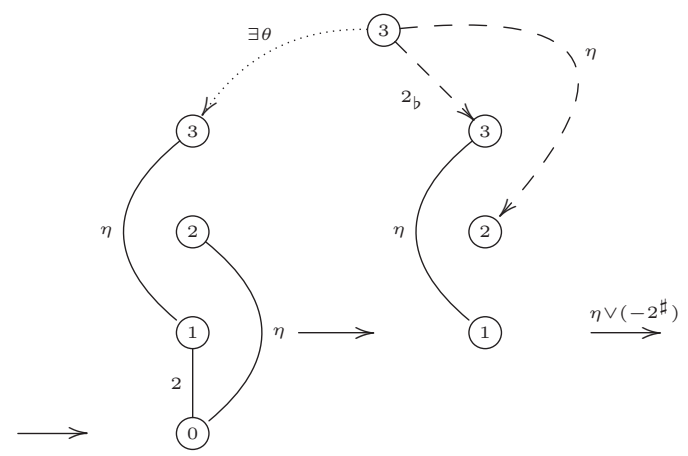

This shows the existence of a map $\theta$ which is well-defined up to indeterminacy which 
lies in the image of

$$
\pi_{3}\left(S^{0}\right) /\left[\eta \pi_{3}\left(S^{1}\right)+2 \pi_{3}\left(S^{0}\right)\right]=\pi_{3}\left(S^{0}\right) / 2 \pi_{3}\left(S^{0}\right) \cong \mathbb{Z}_{2},
$$

so there are two choices of such a map $\theta$ up to homotopy. Because of the $\eta$ component on the 2-sphere, the mapping cone of $\theta$ has the form

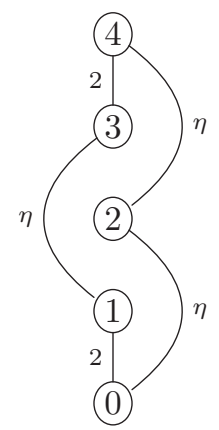

and its cohomology is the Joker $\mathcal{A}(1)$-module. The $\mathcal{A}$-module structure has a $\mathrm{Sq}^{4}$ action between degrees 0 and 4 and this could be zero or non-zero. Each of these possibilities can occur, depending on which of the two of choices for $\theta$ is made. Putting all this together with the algebraic identity (1.1) we obtain the following.

Theorem 4.1. There are two equivalence classes of finite 2-local $C W$ spectra, $J_{0}$ and $J_{1}$, whose cohomology realise the $\mathcal{A}$-modules Joker $_{0}$ and Joker $_{1}$. Up to suspension, $J_{0}$ and $J_{1}$ are Spanier-Whitehead dual, i.e.,

$$
D J_{0} \simeq \Sigma^{-4} J_{1} .
$$

Up to degree 12, the Adams $\mathrm{E}_{2}$-terms for such Joker spectra are almost identical, differing only by an $h_{0}$ multiplication in the 6-column and having no non-trivial differentials.

Here is a useful consequence of the existence of such Joker spectra; we assume this was known to Mark Mahowald but have not been able to locate an explicit statement on the existence of Joker spectra in his published work - however, see Remark 4.3 and also [11, Section 1.7].

Corollary 4.2. The (-1)-connected cover of $k \mathrm{O}$ satisfies

$$
k \mathrm{O} \wedge \Sigma^{2} J_{0} \sim k \mathrm{O}\langle 2\rangle \sim k \mathrm{O} \wedge \Sigma^{2} J_{1} .
$$

It is well known that the other spectra which appear in the Whitehead tower of $k \mathrm{O}$ can all be defined in terms of $k \mathrm{O}$-module spectra of the form $k \mathrm{O} \wedge W$ where the $\mathcal{A}(1)^{*}$-module $H^{*}(W)$ has one of the following forms.

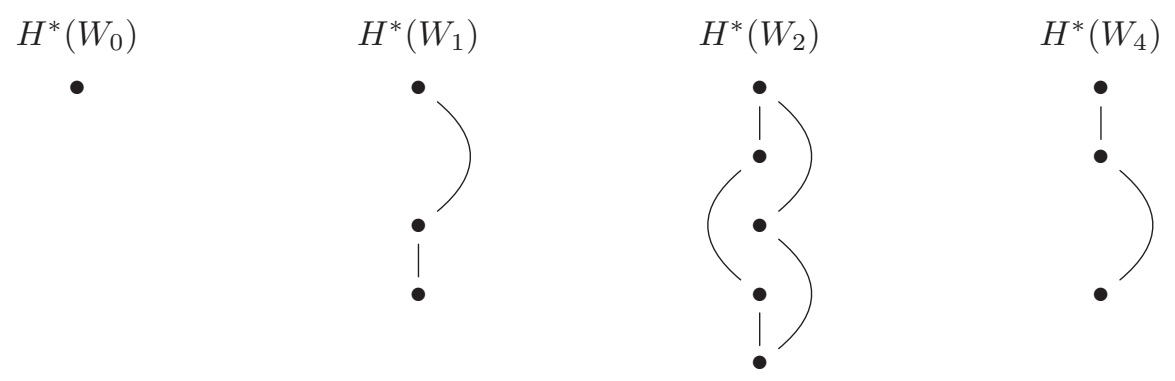


In general, when $r=0,1,2,4$ and $m \geqslant 0$,

$$
k \mathrm{O}\langle 8 m+r\rangle \sim k \mathrm{O} \wedge \Sigma^{8 m} W_{r} .
$$

For more details see $[\mathbf{1 2}, \mathbf{1 3}]$.

Remark 4.3. A spectrum whose cohomology agrees with $\mathcal{A}(1)$ as an $\mathcal{A}(1)$-module (referred to as a 'space' in [13, Remark 1.6]) can be constructed using our Joker spectra. The following construction makes use of detailed information on homotopy groups that can be read off from Adams spectral sequence charts. Starting with $J$ being either of $J_{0}$ or $J_{1}$ we find that there is a generator $u$ of $\pi_{2}(J) \cong \mathbb{Z}_{(2)}$ in Adams filtration 1 (this is a manifestation of $v_{1}$ and has degree 2 on the 2-cell). Also, $\eta u=$ 0 so $u$ extends to a map $S^{2} \cup_{\eta} e^{4} \rightarrow J$. As $\pi_{4}(J)=0$, this also extends to a map $f: S^{2} \cup_{\eta} e^{4} \cup_{2} e^{3} \rightarrow J$ where the attaching map of the 3-cell is yet another avatar of $v_{1}$. The cohomology of the mapping cone $C(f)$ has basis elements in the same degrees as $\mathcal{A}(1)$ and all but one Steenrod operation (indicated by the dashed line below) are clear from the above description.

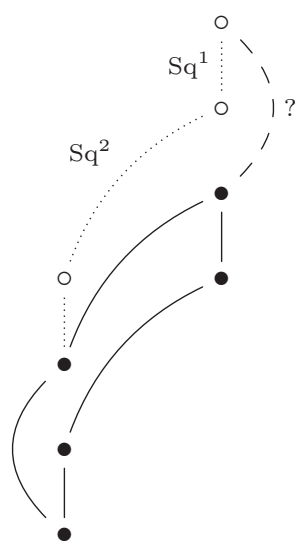

The relation $\mathrm{Sq}^{2} \mathrm{Sq}^{2}=\mathrm{Sq}^{1} \mathrm{Sq}^{2} \mathrm{Sq}^{1}$ shows that this is indeed a $\mathrm{Sq}^{2}$ and therefore as $\mathcal{A}(1)$-modules, $H^{*}(C(f)) \cong \mathcal{A}(1)$.

Of course, the action of $\mathcal{A}$ on $H^{*}(J)$ extends to one on $H^{*}(C(f))$, thus giving at least two different $\mathcal{A}$-module structures on $\mathcal{A}(1)$. In [9, Theorem 1.4], Davis and Mahowald gave a different construction realising all four of the possible $\mathcal{A}$-module structures known to exist.

For small $n$ we can realise $\operatorname{Joker}(n)_{0}$ and $\operatorname{Joker}(n)_{1}$ as the cohomology of spectra.

Theorem 4.4. For $n=2,3$ there are finite 2-local $C W$ spectra, $J(n)_{0}$ and $J(n)_{1}$, whose cohomology restricts to $\mathcal{A}(n)$-modules isomorphic to $\operatorname{Joker}(n)$. These realise the two $\mathcal{A}$-module structures extending the two dual $\mathcal{A}(n)$-module structures. Up to suspension, $J(n)_{1}$ can be taken to be the Spanier-Whitehead dual of $J(n)_{0}$.

Proof. The approach of Section 4 also works using the Toda brackets $\langle\eta, \nu, \eta\rangle$ and $\langle\nu, \sigma, \nu\rangle$ given in (3.1). 
The case $n=2$ :

Using ideas and notation from Section 3 we can form a map

$$
S^{3} \vee C(\Sigma \nu) \stackrel{\nu \vee\left(-\eta^{\sharp}\right)}{\longrightarrow} S^{0},
$$

whose mapping cone fits into a cofibre sequence

$$
S^{0} \rightarrow C\left(\nu \vee\left(-\eta^{\sharp}\right)\right) \rightarrow S^{4} \vee C\left(\Sigma^{2} \nu\right) \rightarrow S^{1} .
$$

The map

$$
S^{7} \stackrel{\Sigma^{4} \nu \vee\left(\Sigma^{5} \eta\right)_{b}}{\longrightarrow} S^{4} \vee C\left(\Sigma^{2} \nu\right)
$$

projects to

$$
\left(\Sigma \nu^{2}-\eta^{\sharp}\left(\Sigma^{5} \eta\right)_{b}\right): S^{7} \rightarrow S^{1},
$$

which is null homotopic as $\langle\eta, \nu, \eta\rangle=\left\{\nu^{2}\right\}$. Hence $\Sigma^{4} \nu \vee\left(\Sigma^{5} \eta\right)_{b}$ factors through a map

$$
\theta^{\prime}: S^{7} \rightarrow C\left(\nu \vee\left(-\eta^{\sharp}\right)\right),
$$

whose mapping cone has the following form.

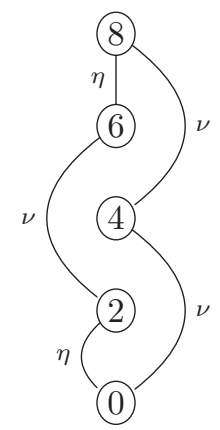

The indeterminacy in $\theta^{\prime}$ is

$$
\pi_{7}\left(S^{0}\right) /\left[\nu \pi_{4}\left(S^{0}\right)+\eta \pi_{6}\left(S^{0}\right)\right]=\pi_{7}\left(S^{0}\right) /\{0\} \cong \mathbb{Z}_{8}
$$

The case $n=3$ :

A similar argument works and we obtain the desired spectrum as the mapping cone of a map

$$
\theta^{\prime \prime}: S^{15} \rightarrow C\left(\sigma \vee\left(-\nu^{\sharp}\right)\right)
$$

The indeterminacy in $\theta^{\prime \prime}$ is

$$
\pi_{15}\left(S^{0}\right) /\left[\sigma \pi_{8}\left(S^{0}\right)+\nu \pi_{12}\left(S^{0}\right)\right]=\pi_{15}\left(S^{0}\right) /\{0\} \cong \mathbb{Z}_{32} .
$$

Remark 4.5. In similar fashion to the construction of a realisation of $\mathcal{A}(1)$ described in Remark 4.3, we can use either of the spectra $J(2)_{0}$ or $J(2)_{1}$ to build a spectrum whose cohomology realises the double $\Delta \mathcal{A}(1)$. In [15], such a spectrum is denoted $D \mathcal{A}(1)$, but this clashes with standard notation for Spanier-Whitehead duals so we avoid using it here. We sketch the details, making use of the information that can be read off of Adams spectral sequence charts. 
Choose $J(2)$ to be either $J(2)_{0}$ or $J(2)_{1}$. We start with a map $S^{5} \rightarrow J(2)$ realising the generator of $\pi_{5}(J(2)) \cong \mathbb{Z}_{2}$ (this has Adams filtration 1); since $\nu \pi_{5}(J(2))=\{0\}$, this extends to a map $S^{5} \cup_{\nu} e^{9} \rightarrow J(2)$. As $\pi_{10}(J(2))=\{0\}$ there is an extension to a map $g: S^{5} \cup_{\nu} e^{9} \cup_{\eta} e^{11} \rightarrow J(2)$ and the cohomology of its mapping cone has the following form where short/long lines indicate $\mathrm{Sq}^{2} / \mathrm{Sq}^{4}$ actions.

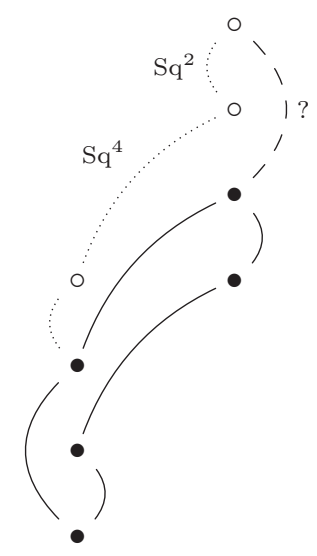

Using the type (B) Wall relation [18] we see that $\mathrm{Sq}^{4} \mathrm{Sq}^{4}+\mathrm{Sq}^{2} \mathrm{Sq}^{4} \mathrm{Sq}^{2}$ acts trivially on Joker(2) and so the dashed line must be a non-trivial $\mathrm{Sq}^{4}$ action. Therefore $H^{*}(C(g)) \cong \Delta \mathcal{A}(1)$ as $\mathcal{A}(2)$-modules. Of course there are two possible $\mathcal{A}$ actions depending on which choice of $J(2)$ we make giving different $\mathrm{Sq}^{16}$ actions.

An attempt at a direct analogue of the preceding argument for the next case runs into difficulties as $\pi_{18}(J(3)) \neq\{0\}$ when $J(3)$ is either of $J(3)_{0}$ or $J(3)_{1}$.

In the other direction we have some non-existence results.

Theorem 4.6. For $n \geqslant 4$, there is no finite 2-local $C W$ spectrum whose cohomology restricts to an $\mathcal{A}(n)$-module isomorphic to $\operatorname{Joker}(n)$.

When $n \geqslant 5$ such a spectrum would violate Adams' Hopf invariant 1 theorem because of the large gap between the two elements of lowest degrees. However, in all cases we can use the precise statement of the following crucial result on the factorisation of primary operations. Here $X$ is a connective spectrum and we explain the notation after the statement.

Theorem 4.7 (Adams [1, Theorem 4.6.1]). Let $k \geqslant 3$ and suppose that $u \in H^{m}(X)$ for $m>0$ satisfies $\mathrm{Sq}^{2^{r}} u=0$ for $0 \leqslant r \leqslant k$. Then

$$
\mathrm{Sq}^{2^{k+1}} u \equiv \sum_{\substack{0 \leqslant i \leqslant j \leqslant k \\ j \neq i+1}} \alpha_{i, j, k} \Phi_{i, j} u \quad \text { (mod indeterminacy). }
$$

In this result, the secondary operation $\Phi_{i, j}$ has degree $2^{i}+2^{j}-1$, and the primary operation $\alpha_{i, j, k} \in \mathcal{A}$ has degree $2^{k+1}-2^{i}-2^{j}+1$. The indeterminacy is the sum of 
the indeterminacies of all the $\Phi_{i, j}$ appearing and has form

$$
\sum_{\substack{0 \leqslant i \leqslant j \leqslant k \\ j \neq i+1}} \alpha_{i, j, k} Q^{*}(X ; i, j),
$$

for certain subgroups $Q^{*}(X ; i, j) \subseteq H^{*}(X)$. Finally, for each pair $i, j$ occurring,

$$
1 \leqslant \operatorname{deg} \alpha_{i, j, k} \leqslant 2^{k+1}-1 \text {. }
$$

Proof of Theorem 4.6. Let $n \geqslant 4$ and suppose that a Joker spectrum $J$ exists for this $n$.

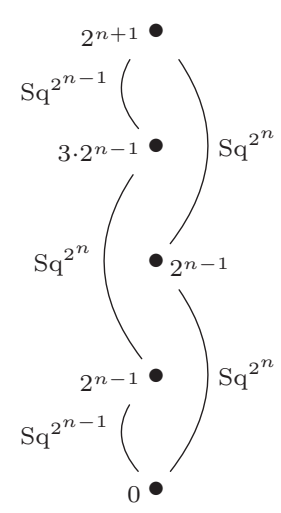

Consider the non-zero element $u$ in degree $2^{n-1}$. Taking $k=n-1$, we can apply Theorem 4.7. Carefully examining the possible terms in the sum we find that they are all 0 , and similarly so is the indeterminacy. The conclusion is that $\operatorname{Sq}^{2^{n}} u=0$, contradicting the assumptions on $J$.

\section{Some unstable realisations}

Now we turn to the question of unstable realisations, i.e., as the cohomology of spaces. If $X$ is a 2-local space whose cohomology $\widetilde{H}^{*}(X)$ is isomorphic to Joker* $[n]$ as an $\mathcal{A}(1)$-module then $n \geqslant 2$ since $\mathrm{Sq}^{2}$ acts non-trivially on the bottom generator. Similarly, realising the $\mathcal{A}$-module $\operatorname{Joker}_{1}[n]$ unstably requires that $n \geqslant 4$.

Theorem 5.1. There are finite 2-local $C W$ complexes $X_{2}$ and $X_{4}$ such that as $\mathcal{A}$ modules,

$$
\widetilde{H}^{*}\left(X_{2}\right) \cong \operatorname{Joker}_{0}^{*}[2], \quad \widetilde{H}^{*}\left(X_{4}\right) \cong \operatorname{Joker}_{1}^{*}[4] .
$$

Proof. Corollary 4.2 suggests looking for an unstable realisation of the Joker in the

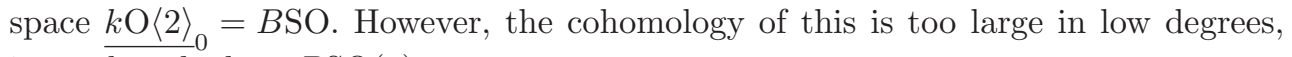
instead we look at $B \mathrm{SO}(3)$.

Recall the $\mathrm{Wu}$ formula

$$
\mathrm{Sq}^{r} w_{m}=w_{r} w_{m}+\sum_{1 \leqslant i \leqslant r}\left(\begin{array}{c}
r-m \\
i
\end{array}\right) w_{r-i} w_{m+i} .
$$

Using this, in $H^{*}(B \mathrm{SO}(3))=\mathbb{F}_{2}\left[w_{2}, w_{3}\right]$ we obtain

$$
\mathrm{Sq}^{1} w_{2}=w_{3}, \quad \mathrm{Sq}^{2} w_{3}=0 .
$$


Thus we obtain a copy of the Joker in the $\mathcal{A}(1)$-module $H^{*}(B \mathrm{SO}(3))$.

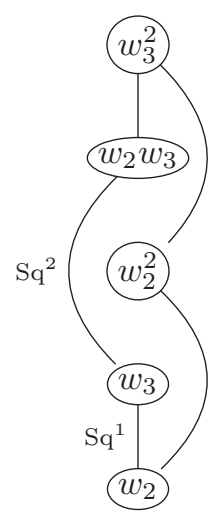

However, $H^{6}(B \mathrm{SO}(3))=\mathbb{F}_{2}\left\{w_{3}^{2}, w_{2}^{3}\right\}$, so we next remove the additional generator by considering the fibre of the map classifying $w_{2}^{3}$,

$$
B \mathrm{SO}(3) \stackrel{w_{2}^{3}}{\longrightarrow} K\left(\mathbb{F}_{2}, 6\right),
$$

which we will denote by $B \mathrm{SO}(3)\left\{w_{2}^{3}\right\}$. Calculating $H^{*}\left(B \mathrm{SO}(3)\left\{w_{2}^{3}\right\}\right)$ using the Serre spectral sequence or the Eilenberg-Moore spectral sequence we find that when $k \leqslant 6$,

$$
H^{k}\left(B \operatorname{SO}(3)\left\{w_{2}^{3}\right\}\right) \cong\left(\mathbb{F}_{2}\left[w_{2}, w_{3}\right] /\left(w_{2}^{3}\right)\right)^{k},
$$

so taking the 6 -skeleton of a minimal CW realisation (in the sense of [3, Section 3] for example) we obtain an isomorphism of $\mathcal{A}(1)$-modules

$$
H^{*}\left(B \mathrm{SO}(3)\left\{w_{2}^{3}\right\}^{[6]}\right) \cong \operatorname{Joker}_{0}[2] .
$$

To realise Joker ${ }_{1}$ [4], we start with an unstable complex $S^{3} \cup_{\eta_{3}} e^{5} \cup_{2} e^{6}$ which exists since the suspension of the Hopf map $S^{3} \rightarrow S^{2}$ gives an element $\eta_{3} \in \pi_{4}\left(S^{3}\right)$ of order 2 , see $\left[\mathbf{1 7}\right.$, Chapter V]. Smashing with the Moore space $S^{1} \cup_{2} e^{2}$ we obtain a CW complex $X^{\prime}$ whose cohomology as an $\mathcal{A}(1)$-module realises the 4 -fold suspensions of the 'whiskered Joker' module

$$
\text { Joker }^{\prime}=\mathcal{A}(1) / \mathcal{A}(1)\left\{\mathrm{Sq}^{2} \mathrm{Sq}^{1} \mathrm{Sq}^{2}\right\}=\mathcal{A}(1) / \mathcal{A}(1)\left\{\mathrm{Sq}^{2} \mathrm{Sq}^{3}\right\}
$$

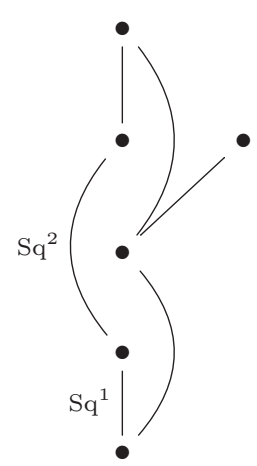

Labelling cells and cohomology generators in the obvious way, $X^{\prime}$ has the following cell diagram. 

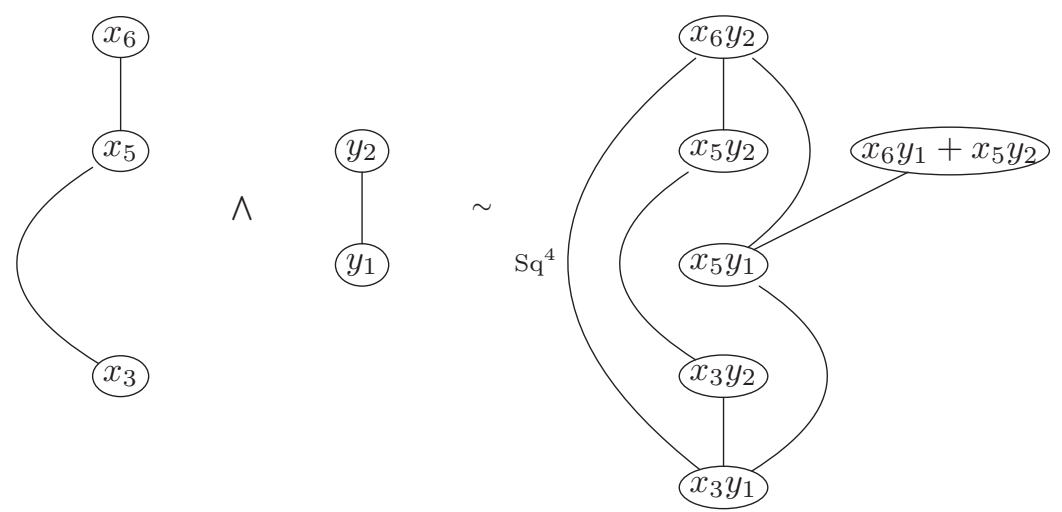

Notice that as well as the $\mathrm{Sq}^{1}$ and $\mathrm{Sq}^{2}$ actions we also have $\mathrm{Sq}^{4}\left(x_{3} y_{1}\right)=x_{6} y_{2}$ so this agrees with Joker $_{1}^{\prime}[4]$ as an $\mathcal{A}$-module.

We will begin by showing that there is a factorisation

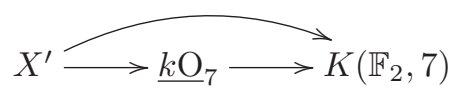

of the map classifying $x_{6} y_{1}+x_{5} y_{2} \in H^{7}\left(X^{\prime}\right)$. We will do this by producing a map of spectra $\Sigma^{\infty} X^{\prime} \rightarrow \Sigma^{7} k \mathrm{O}$ by dualising a map

$$
S^{1} \rightarrow k \mathrm{O} \wedge \Sigma^{8} D \Sigma^{\infty} X^{\prime} \sim k \mathrm{O} \wedge W^{\prime \prime},
$$

where $D$ denotes Spanier-Whitehead dual and $W^{\prime \prime}$ is a CW spectrum whose cohomology realises the dual whiskered Joker $\mathcal{A}(1)$-module Joker $_{1}^{\prime \prime}=$ DJoker $_{1}^{\prime}$ shown in the following diagram.

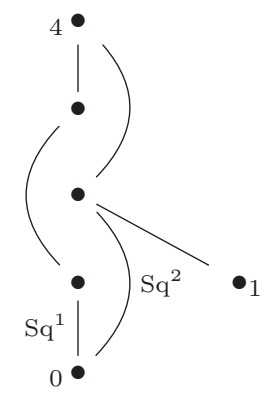

Since we are interested in elements of $\pi_{1}\left(k \mathrm{O} \wedge W^{\prime \prime}\right)$, we need to consider the $t-s=1$ column in the Adams spectral sequence

$$
\mathrm{E}_{2}^{s, t}=\operatorname{Ext}_{\mathcal{A}}^{s, t}\left(H^{*}\left(k \mathrm{O} \wedge W^{\prime \prime}\right), \mathbb{F}_{2}\right) \cong \operatorname{Ext}_{\mathcal{A}(1)}^{s, t}\left(H^{*}\left(W^{\prime \prime}\right), \mathbb{F}_{2}\right) \Longrightarrow \pi_{t-s}\left(k \mathrm{O} \wedge W^{\prime \prime}\right)
$$

and a portion of the $\mathrm{E}_{2}$-term is shown in Figure 1 . As the generator in $\mathrm{E}_{2}^{0,1}$ cannot support a differential there is a non-trivial element of $\pi_{1}\left(k \mathrm{O} \wedge W^{\prime \prime}\right)$ detected in the zero line by the only $\mathcal{A}(1)$-indecomposable element of $H^{1}\left(W^{\prime \prime}\right)$. Hence there is a dual element of $k \mathrm{O}^{7}\left(X^{\prime}\right)$ with the desired properties.

Now take a minimal CW complex equivalent to the fibre of the map $X^{\prime} \rightarrow \underline{k \mathrm{O}_{7}}$ and let $X_{4}$ be its 8 -skeleton. By a straightforward calculation with either of the 
Serre or Eilenberg-Moore spectral sequences and making use of the $k \mathrm{O}$ results of Examples A.3, we find that $H^{*}(X)$ realises the $\mathcal{A}$-module $\mathrm{Joker}_{1}[4]$.

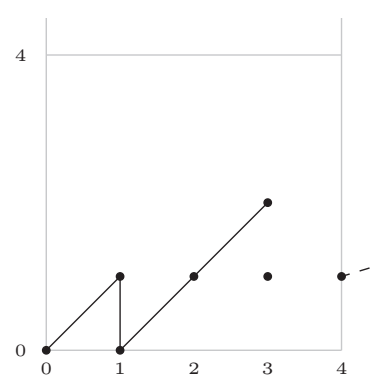

Figure 1: $\operatorname{Ext}_{\mathcal{A}(1)}^{s, t}\left(\mathrm{Joker}^{\prime \prime}, \mathbb{F}_{2}\right): 0 \leqslant s \leqslant 4$ and $0 \leqslant t-s \leqslant 4$.

Theorem 5.2. There are finite 2-local $C W$ complexes $Y_{4}$ and $Y_{8}$ such that as $\mathcal{A}$ modules,

$$
\widetilde{H}^{*}\left(Y_{4}\right) \cong \operatorname{Joker}(2)_{0}^{*}[4], \quad \widetilde{H}^{*}\left(Y_{8}\right) \cong \operatorname{Joker}(2)_{1}^{*}[8] .
$$

Proof. A similar construction to that of $X_{2}$ starting with $B S U(3)$ leads to an unstable realisation of Joker $(2)_{0}[4]$.

We will realise Joker $(2)_{1}[8]$ using a similar approach to that for $X_{4}$. By Toda $[\mathbf{1 7}$, Proposition 5.8], $\pi_{10}\left(S^{6}\right)$ is trivial, so the suspensions of the Hopf maps give elements $\eta_{9} \in \pi_{10}\left(S^{9}\right)$ and $\nu_{6} \in \pi_{9}\left(S^{6}\right)$ which satisfy

$$
0=\nu_{6} \circ \eta_{9} \in \pi_{10}\left(S^{6}\right) \text {. }
$$

Hence we can form $S^{5} \cup_{\nu_{5}} e^{9} \cup_{\eta_{9}} e^{11}$ and $S^{3} \cup_{\eta_{3}} e^{5}$. By smashing these together we obtain a CW complex

$$
Y^{\prime}=\left(S^{5} \cup_{\nu_{5}} e^{9} \cup_{\eta_{9}} e^{11}\right) \wedge\left(S^{3} \cup_{\eta_{3}} e^{5}\right),
$$

whose cohomology realises the $\mathcal{A}$-module with non-trivial $\mathrm{Sq}^{8}$-action and is the 8 -fold suspension of the whiskered double Joker cyclic $\mathcal{A}(2)$-module

$$
\operatorname{Joker}(2)_{1}^{\prime}=\mathcal{A}(2) / \mathcal{A}(2)\left\{\mathrm{P}_{1}^{0}, \mathrm{P}_{1}^{1}, \mathrm{P}_{2}^{1}, \mathrm{Sq}^{4} \mathrm{Sq}^{6}\right\} \text {. }
$$

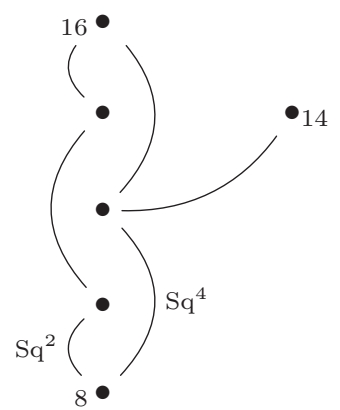

We would like to define a map $Y^{\prime} \rightarrow \underline{\operatorname{tmf}}_{14}$ so that the cohomology class in $H^{14}\left(\underline{\operatorname{tmf}}_{14}\right)$ 
carried on the bottom cell is mapped to $\mathrm{Sq}^{2} \mathrm{Sq}^{4} y_{8}$ by the induced homomorphism, where $y_{8} \in H^{8}(Y)$ is the generator. Such a map corresponds to a map of spectra $\Sigma^{\infty} Y^{\prime} \rightarrow \Sigma^{14}$ tmf or equivalently a map

$$
S^{0} \rightarrow \Sigma^{14}\left(D \Sigma^{\infty} Y^{\prime}\right) \wedge \operatorname{tmf} \sim \Sigma^{-2} Z^{\prime \prime} \wedge \operatorname{tmf},
$$

where $Z^{\prime \prime}$ is a CW spectrum whose cohomology realises the other whiskered double Joker $\mathcal{A}(2)$-module Joker $(2)_{1}^{\prime \prime}$ shown in the following diagram.

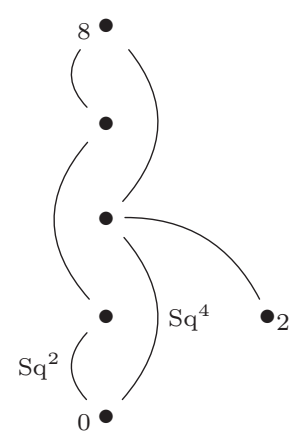

Since we are interested in elements of $\pi_{2}\left(\operatorname{tmf} \wedge Z^{\prime \prime}\right)$, we need to consider the $t-s=2$ column in the Adams spectral sequence

$$
\mathrm{E}_{2}^{s, t}=\operatorname{Ext}_{\mathcal{A}}^{s, t}\left(H^{*}\left(\operatorname{tmf} \wedge Z^{\prime \prime}\right), \mathbb{F}_{2}\right) \cong \operatorname{Ext}_{\mathcal{A}(2)}^{s, t}\left(H^{*}\left(Z^{\prime \prime}\right), \mathbb{F}_{2}\right) \Longrightarrow \pi_{t-s}\left(\operatorname{tmf} \wedge Z^{\prime \prime}\right)
$$

and a portion of the $\mathrm{E}_{2}$-term is shown in Figure 2. As the generator in $\mathrm{E}_{2}^{0,2}$ cannot support a differential this shows that there is a suitable element of $\pi_{2}\left(\operatorname{tmf} \wedge Z^{\prime \prime}\right)$ and hence of $\operatorname{tmf}^{14}\left(Y^{\prime}\right)$.

Now consider the fibre of the above map $Y^{\prime} \rightarrow \underline{\operatorname{tmf}}_{14}$. By a spectral sequence calculation and making use of the tmf results of Examples A.3 we see that its cohomology agrees with $\operatorname{Joker}(2)_{1}[8]$ up to degree 21 . The 16 -skeleton of a minimal CW realisation of this fibre is a $\mathrm{CW}$ complex $Y_{8}$ whose cohomology as an $\mathcal{A}$-module agrees with $\operatorname{Joker}(2)_{1}[8]$.

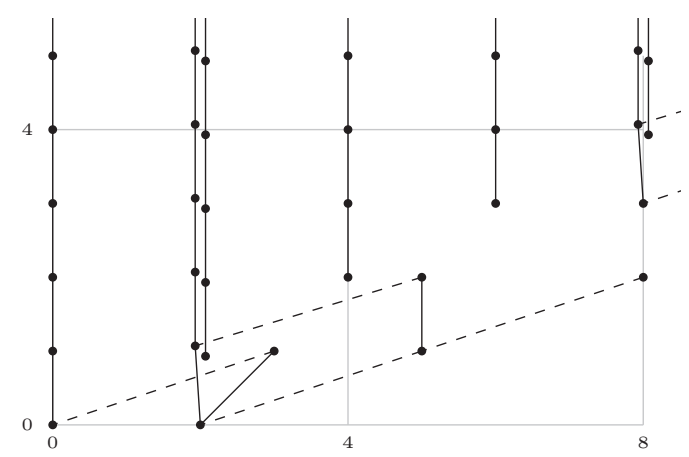

Figure 2: $\operatorname{Ext}_{\mathcal{A}(2)}^{s, t}\left(\operatorname{Joker}(2)^{\prime \prime}, \mathbb{F}_{2}\right): 0 \leqslant s \leqslant 5$ and $0 \leqslant t-s \leqslant 8$. 
It is unclear how to realise $\operatorname{Joker}(3)_{0}[8]$ since there is no obvious analogue of $B \mathrm{SO}(3)$ and $B \mathrm{SU}(3)$ which appears relevant. Similarly, our argument for $X_{4}$ and $Y_{8}$ has no obvious generalisation since because of the non-existence of suitable elements of Hopf invariant 1 there is no spectrum playing an analogous rôle to $k \mathrm{O}$ and tmf in the last steps.

\section{Concluding remarks}

The appearance of the elements of Kervaire invariant 1 in our realisations of Joker modules raises the question of whether there other $\mathcal{A}(n)$-modules which admit realisations when $\theta_{n}$ exists, i.e., when $n=4,5$ and possibly 6 . In particular, by [20, Theorem 5.2],

$$
\left\{\theta_{4}\right\}=\left\langle 2, \sigma^{2}+\kappa, 2 \sigma, \sigma\right\rangle,
$$

while older work of Barratt, Mahowald and Tangora, and Kochman shows that

$$
\left\{\theta_{4}\right\}=\left\langle 2, \sigma^{2}, 2, \sigma^{2}\right\rangle=\left\langle 2, \sigma^{2}, \sigma^{2}, 2\right\rangle=\langle 2 \sigma, \sigma, 2 \sigma, \sigma\rangle=\left\langle 2, \sigma^{2}, 2 \sigma, \sigma\right\rangle .
$$

These suggest the intriguing possibility that appropriate constructions associated with such 4 -fold Toda brackets might lead to realisation results for some interesting $\mathcal{A}(4)$-modules.

\section{Appendix A. Some connectivity results}

Let $p$ be a prime and let $f: X \rightarrow Y$ be a map between two finite type $p$-local connective spectra or spaces which are simply connected or at least have abelian fundamental groups.

Recall that $f$ is called an $n$-equivalence if $f_{*}: \pi_{k}(X) \rightarrow \pi_{k}(Y)$ is an isomorphism for $k<n$ and an epimorphism if $k=n$; this is equivalent to the mapping cone $C_{f}$ being $n$-connected. It is well-known that the following are also equivalent conditions:

- $f_{*}: H_{k}\left(X ; \mathbb{Z}_{(p)}\right) \rightarrow H_{k}\left(Y ; \mathbb{Z}_{(p)}\right)$ is an isomorphism if $k<n$ and an epimorphism if $k=n$.

- $f_{*}: H_{k}\left(X ; \mathbb{F}_{p}\right) \rightarrow H_{k}\left(Y ; \mathbb{F}_{p}\right)$ is an isomorphism if $k<n$ and an epimorphism if $k=n$.

The next result relates connectivity information for spectra and their associated infinite loop spaces. Although such results are undoubtedly standard we are not aware of convenient references and we use them to establish Examples A.3.

We will denote the $m$-th space in a spectrum $X$ by $\underline{X}_{m}=\Omega^{\infty} \Sigma^{m} X$. If $f: X \rightarrow Y$ is a map of $(-1)$-connected spectra then for each $m \geqslant 0$ there is an induced infinite loop map $f_{m}: \underline{X}_{m} \rightarrow \underline{Y}_{m}$.

Lemma A.1. Let $f: X \rightarrow Y$ be an n-equivalence. Then for each $m \geqslant 1, f_{m}: \underline{X}_{m} \rightarrow$ $\underline{Y}_{m}$ is an $m+n$-equivalence, hence $\left(f_{m}\right)_{*}: H_{k}\left(\underline{X}_{m} ; \mathbb{F}_{p}\right) \rightarrow H_{k}\left(\underline{Y}_{m} ; \mathbb{F}_{p}\right)$ is an isomorphism if $k<m+n$ and an epimorphism if $k=m+n$.

Here is a sample application; we only state this for the prime 2, but a similar result also holds for odd primes. 
Corollary A.2. Take $p=2$ and let $X$ be a (-1)-connected spectrum and suppose that $\pi_{0}(X)$ is a cyclic $\mathbb{Z}_{(2)}$-module with generator given by a map $j: S^{0} \rightarrow X$. If $j$ is an $n$-equivalence then for each $m>n$, and $m<k \leqslant m+n$,

$$
H_{k}\left(\underline{X}_{m} ; \mathbb{F}_{2}\right)=0 \text {. }
$$

Proof. Recall that for $m \geqslant 1$, the homology of $\underline{S}_{m}^{0}=\mathrm{Q} S^{m}$ is given by

$$
H_{*}\left(\mathrm{Q} S^{m} ; \mathbb{F}_{2}\right)=\mathbb{F}_{2}\left[\mathrm{Q}^{I} x_{m}: I \text { admissible, } \operatorname{exc}(I)>m\right],
$$

where $x_{m} \in H_{m}\left(\mathrm{Q} S^{m} ; \mathbb{F}_{2}\right)$. Thus the three elements of lowest positive degree are $x_{m}$, $x_{m}^{2}$ and $\mathrm{Q}^{2 m+1} x_{m}$ in degrees $m, 2 m>m+n$ and $2 m+1>m+n$ respectively.

The infinite loop map $j_{m}$ induces an algebra homomorphism $\left(j_{m}\right)_{*}$ over the DyerLashof algebra. By assumption on $j$,

$$
\left(j_{m}\right)_{*}: H_{k}\left(\mathrm{Q} S^{m} ; \mathbb{F}_{2}\right) \rightarrow H_{k}\left(\underline{X}_{m} ; \mathbb{F}_{2}\right)
$$

is an isomorphism when $k<m+n$ and an epimorphism when $k=m+n$.

Thus the lowest degree non-zero element of $H_{*}\left(\underline{X}_{m} ; \mathbb{F}_{2}\right)$ not in the image of $\left(j_{m}\right)_{*}$ occurs in some degree $k_{0} \geqslant m+n+1$ where $k_{0}-m$ is also the smallest degree for which

$$
\operatorname{coker}\left[j_{*}: H_{k}\left(S^{0} ; \mathbb{F}_{2}\right) \rightarrow H_{k}\left(X ; \mathbb{F}_{2}\right)\right] \neq 0
$$

Examples A.3. We set $H_{*}(-)=H_{*}\left(-; \mathbb{F}_{2}\right)$.

Recall that

$$
H_{*}(k \mathrm{O})=\mathbb{F}_{2}\left[\zeta_{1}^{4}, \zeta_{2}^{2}, \zeta_{3}, \ldots\right] \subseteq \mathcal{A}_{*}, \quad H_{*}(\operatorname{tmf})=\mathbb{F}_{2}\left[\zeta_{1}^{8}, \zeta_{2}^{4}, \zeta_{3}^{2}, \zeta_{4}, \ldots\right] \subseteq \mathcal{A}_{*} .
$$

Thus on $H_{*}(-)$ the units $j^{k \mathrm{O}}: S \rightarrow k \mathrm{O}$ and $j^{\text {tmf }}: S \rightarrow$ tmf induce homomorphisms whose cokernels coker $j_{*}^{k \mathrm{O}}$ and coker $j_{*}^{k \mathrm{O}}$ have non-zero elements of lowest degrees 4 and 8 respectively. Thus $j^{k \mathrm{O}}$ is a 3 -equivalence and $j^{\mathrm{tmf}}$ is a 7 -equivalence.

For $4 \leqslant m<k \leqslant m+3$,

$$
H_{k}\left(\underline{k \mathrm{O}}_{m}\right)=0
$$

while for $8 \leqslant m<k \leqslant m+7$,

$$
H_{k}\left(\underline{\operatorname{tmf}}_{m}\right)=0 \text {. }
$$

The results for $k \mathrm{O}$ can also be deduced from work of Dena Cowen Morton [8] but as far as we know the algebra structure of the Hopf ring for tmf has not been determined.

\section{References}

[1] J.F. Adams, On the non-existence of elements of Hopf invariant one, Ann. of Math. (2) 72 (1960), 20-104.

[2] J.F. Adams and S.B. Priddy, Uniqueness of BSO, Math. Proc. Cambridge Philos. Soc. 80 (1976), 475-509.

[3] A.J. Baker and J.P. May, Minimal atomic complexes, Topology 43 (2004), 645-665. 
[4] P. Bhattacharya and N. Ricka, The stable Picard group of $\mathcal{A}(2)$ (2017), available at arXiv: 1702.01493.

[5] R.R. Bruner and J.P.C. Greenlees, Connective Real K-Theory of Finite Groups, Math. Surveys Monogr., vol. 169, American Mathematical Society, 2010.

[6] M. Catanzaro, Finitely Presented Modules over the Steenrod Algebra in Sage, MA thesis, Wayne State University, 2011, http://www . math.wayne.edu/ mike/ mods/Essayfinal.pdf.

[7] J.M. Cohen, The decomposition of stable homotopy, Ann. of Math. (2) 87 (1968), 305-320.

[8] D.S. Cowen Morton, The Hopf ring for bo and its connective covers, J. Pure Appl. Algebra 210 (2007), 219-247.

[9] D.M. Davis and M. Mahowald, $v_{1}$ and $v_{2}$-periodicity in stable homotopy theory, Amer. J. Math. 103 (1981), 615-659.

[10] V. Giambalvo and D.J. Pengelley, The homology of MSpin, Math. Proc. Cambridge Philos. Soc. 95 (1984), no. 3, 427-436.

[11] M.J. Hopkins, Some Problems in Topology, PhD thesis, University of Oxford, 1984.

[12] M. Mahowald, bo-resolutions, Pac. J. Math. 92 (1981), 365-383.

[13] M. Mahowald and R.J. Milgram, Operations which detect $\mathrm{Sq}^{4}$ in connective $K$ theory and their applications, Quart. J. Math. Oxford Ser. (2) 27 (1976), no. 108, 415-432.

[14] H.R. Margolis, Spectra and the Steenrod Algebra: Modules over the Steenrod Algebra and the Stable Homotopy Category, North-Holland, 1983.

[15] A. Mathew, The homology of tmf, Homology Homotopy Appl. 18 (2016), 1-29.

[16] R.E. Mosher and M.C. Tangora, Cohomology Operations and Applications in Homotopy Theory, Harper \& Row, 1968.

[17] H. Toda, Composition Methods in Homotopy Groups of Spheres, Ann. of Math. Stud., vol. 49, Princeton University Press, 1962.

[18] C.T.C. Wall, Generators and relations for the Steenrod algebra, Ann. of Math. 72 (1960), 429-444.

[19] G.W. Whitehead, Recent Advances in Homotopy Theory, CBMS Reg. Conf. Ser. Math., vol. 5, American Mathematical Society, 1970.

[20] Z. Xu, The strong Kervaire invariant problem in dimension 62, Geom. Topol. 20 (2016), 1611-1624.

Andrew Baker a.baker@maths.gla.ac.uk

School of Mathematics \& Statistics, University of Glasgow, Glasgow G12 8QW,

Scotland http://www.maths.gla.ac.uk/ ajb 